\title{
A Silent Myocardial Infarction Diagnosed As an Outdoor Patient: A Case Report
}

\author{
Ibrar $\mathrm{A}^{1^{*}}$, Haris $\mathrm{C}^{2}$, Wafa $\mathrm{S}^{3}$, Muhammad $\mathrm{Z}^{3}$ and Saher $\mathrm{A}^{4}$ \\ ${ }^{1} \mathrm{MBBS}, \mathrm{MD}$, University of Health and Sciences, Pakistan \\ ${ }^{2} \mathrm{MBBS}, \mathrm{MD}$, University of California, Irvine, United States \\ ${ }^{3}$ MBBS, Dow University of Health Sciences, Karachi, Pakistan \\ ${ }^{4} \mathrm{MBBS}, \mathrm{MD}$, Allama Iqbal Medical College, Pakistan
}

${ }^{*}$ Corresponding author: Ibrar A, MBBS, MD, University of Health and Sciences, Pakistan, Tel: 7083402991, E-mail: Ibrar.anjum@gmail.com

Citation: Ibrar A, Haris C, Wafa S, Muhammad Z, Saher A (2018) A Silent Myocardial Infarction Diagnosed As an Outdoor Patient: A Case Report. Saj Case Rep 5: 402

Article history: Received: 11 May 2018, Accepted: 05 September 2018, Published: 07 September 2018

\begin{abstract}
Background: Silent myocardial infarction (SMI) is defined as an Ischemia of the heart in the absence of salient symptoms. We here present a case of silent myocardial infarction (SMI) in a 55-year-old male, with the history of heavy smoking who presented to an outpatient complaining of a headache. He had no history of chest pain, jaw pain, nausea, diaphoresis, or shortness of breath. EKG showed ST-segment elevations (STEMI) in leads V1 to V5 and AVF. Troponin was reactive and raised, and angiogram showed diffuse coronary artery disease. Clinicians should be aware of asymptomatic presentation of myocardial infarction that may be life-threatening cardiac ischemia.
\end{abstract}

Keywords: Silent Heart Attack; Silent MI; Diabetes and MI

\section{Introduction}

Acute coronary syndrome (ACS) is an ambit of heart diseases that include ST-segment, non-ST-segment elevation myocardial infarction, stable and unstable angina [1]. Silent myocardial ischemia (SMI) is defined as the presence of myocardial ischemia in the absence of typical symptoms. The absence of symptoms makes it a diagnostic challenge for a physician resulting in the delay in diagnosis and early intervention [2]. Historically, SMI has been associated with diabetes which is considered an independent risk factor for coronary artery disease [3-5]. However, SMI may also occur in non-diabetic patients [6,7]. Limited data are available on the prevalence of SMI in non-diabetic patients [8]. We here present an unusual case of SMI in a non-diabetic, active smoker to discuss the implications of silent myocardial infarction for the physicians.

\section{Case Presentation}

A 55-year-old male non-diabetic, active smoker with a history of smoking one pack a day for past 30 years presented to outpatient clinic with a presenting complaint of a headache. Headache was of gradual onset, generalized in nature and was present for last twenty four hours. It was mild in intensity but it did not subside by taking Tylenol. On arrival to the outdoor clinic, physical examination revealed that he was hemodynamically stable and afebrile. His blood pressure was 140/90 $\mathrm{mmHg}$. He looked comfortable with no acute distress. There were no signs of shortness of breath and patient sat comfortably on chair. His cardiovascular examination showed a Heart rate of 96 beats per minute and regular rhythm with no murmur, friction or rub. No clinical signs of heart failure were apparent. Chest was clear on auscultation. Neurologic examination was done which revealed no abnormality.

We decided to order electrocardiography (ECG) as a routine investigatory work. ECG showed ST-Segment elevation (STEMI) in leads I, II, V1 to V5 and aFV (Figure 1). Although; the patient denied typical myocardial infarction symptoms like chest pain, nausea, vomiting or shortness of breath. He was admitted to the hospital for further investigation and treatment of a suspected myocardial infarction. Further studies revealed reactive troponin I $5 \mathrm{ng} / \mathrm{ml}$ on the first day of admission (normal <0.5). After 5 hours of admission, CK-MB was $28 \mathrm{U} / \mathrm{I}$, and Creatine phosphokinase was normal at $88 \mathrm{U} / \mathrm{l}$. Further investigation included echocardiogram which showed anterior and inferoseptal wall hypokinesia with ejection fraction (EF) of 40\%-45\%. Patient was 
advices an angiogram which showed diffuse coronary artery disease. Treatment was started with Aspirin (300 mg), Clopidogrel (75 mg), and Metoprolol (25 mg) and patient was advised to follow up in outpatient clinic.

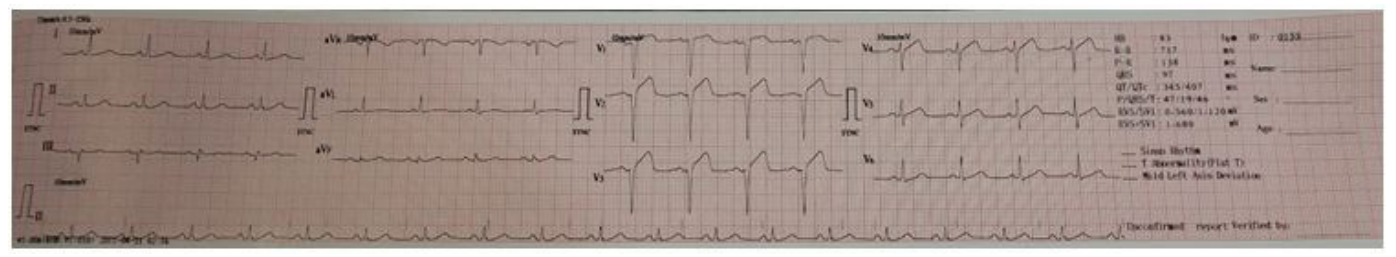

Figure 1: ST segment elevation in leads V1-V5, I, II and aVF

\section{Discussion}

Difference exist in the literature about the incidence, and prognostic significance of SMI which may be partly explained by the differences in the population studied and the investigation of choice by which SMI has been detected (Eg: Q wave in ECG) [9]. Diabetes is the known and accepted comorbidity that increases the prevalence of SMI compared to general population [3]. A study done by Kannel, et al. in which patients were followed for 30 years with a normal ECG and clinical review had shown that almost half of the myocardial infarctions were silent [10]. Cigarette smoking is known to be associated with myocardial infarction and acute coronary events. Why might patients who smoke have an increased prevalence of Silent myocardial infarction (SMI)?

John E, et al. in 1986 studied the effect of smoking a single cigarette on regional myocardial perfusion using positron emission tomography and rubidium-82 (82Rb) [11]. Six of the eight patients with positive exercise test demonstrated a decrease in $82 \mathrm{Rb}$ uptake. This supports the fact that smoking can cause profound silent disturbances of regional myocardial perfusion. Repeated insults of the myocardium due to continuous smoking might represent the underlying mechanism of silent myocardial infarction. As our patient had no apparent signs and symptoms of myocardial infarction and a positive history of heavy smoking, we were compelled to think of a possible association of silent myocardial infarction with smoking.

Cardiac autonomic neuropathy and increase threshold of pain sensitivity are the principal explanations among others. Nicotine activates supraspinal and spinal N-Acetylcholine Receptors (NAChR) which results in opioid and norepinephrine (NEpi) release, resulting in a reduction of descending facilitatory pain pathways and enhances descending inhibitory pain pathways. This mechanism overall reduces transmission of nociceptive input [12]. Cigarette smoking also increases the risk of type 2 diabetes in the general population [13]. Many experimental studies have shown that smoking had adverse effects on the metabolism of glucose and lipids irrespective of diabetes. Cigarette smoking in patients with diabetes was associated with deterioration of metabolic control [14]. This risk may be mediated through direct metabolic effects alone or in combination with a metabolically unfavorable lifestyle resulting in cardiac autonomic neuropathy. Cardiac autonomic neuropathy plays a critical role involving at different levels - from the pain receptors, afferent neurons or gating mechanisms to the supratentorial translation of ischemia into pain. These repeated insults might represent underlying mechanism of silent myocardial infarction linking with smoking. More studies need to be conducted to make smoking as an acceptable cause of SMI.

Other possible risk factors of silent myocardial infarction include increasing age, increased cholesterol levels for a long period of time, high blood pressure, physical inactivity, obesity and psychosocial factors which includes staying aloof and not having healthy relationship with spouse which can ultimately lead to depression; another risk factor for silent MI. People belonging to South Asia and Scandinavia are at a higher risk and males are usually prone to have no apparent symptoms of myocardial infarction according to literature available. It has been found that silent myocardial infarction has a poorer prognosis and such patients are at a higher risk of developing heart failure later in life. Therefore, we urge to carry out ECG of patients who present with any of the abovementioned risk factors on routine basis.

\section{Conclusion}

This case spotlights an essential diagnostic challenge for the general practitioners and cardiologist in early detection of asymptomatic myocardial infarction. Delay in the diagnosis of myocardial infarction results in increased in morbidity and mortality. Clinicians should be aware of the asymptomatic presentation of ischemic heart disease that may be life-threatening. The presence of Silent myocardial infarction (SMI) has significant implications for the patient and physicians should alert actively smoking patients for the possibility of silent ischemia.

\section{References}

1. Amsterdam EA, Wenger NK, Brindis RG, Casey DE, Ganiats TG, et al. (2014) 2014 AHA/ACC Guideline for the Management of Patients with Non-ST-Elevation Acute Coronary Syndromes: a report of the American College of Cardiology/American Heart Association Task Force on Practice Guidelines. J Am Coll Cardiol 23: $139-228$.

2. Ahmed AH, Shankar K, Eftekhari H, MS Munir, Jillian R, et al (2007) Silent myocardial ischemia: Current perspectives and future directions. Exp Clin Cardiol 12: 189-96.

3. Draman MS, Thabit H, Kiernan TJ, O'Neill J, Sreenan S, et al. (2013) A silent myocardial infarction in the diabetes outpatient clinic: case report and review of the literature. Endocrinol Diabetes Metab Case Rep 2013: 130058. 
4. McGill HC, McMahan CA (1998) Determinants of atherosclerosis in the young. Pathobiological Determinants of Atherosclerosis in Youth (PDAY) Research Group. Am J Cardi 82: 30-6.

5. Wilson PW (1998) Diabetes mellitus and coronary heart disease. Am J Kidney Dis 32: 89-100.

6. Chipkin SR, Frid D, Alpert JS, Baker SP, Dalen JE, et al. (1987) Frequency of painless myocardial ischemia during exercise tolerance testing in patients with and without diabetes mellitus. Am J Cardiol 59: 61-5.

7. Cohn PF (1985) Silent myocardial ischemia: classification, prevalence, and prognosis. Am J Med 13: 2-6.

8. Falcone C, Nespoli L, Geroldi D, Gazzaruso C, Buzzi MP, et al. (2003) Silent myocardial ischemia in diabetic and nondiabetic patients with coronary artery disease. Int J Cardiol 90: 219-27.

9. Zhang ZM, Rautaharju PM, Prineas RJ, Rodriguez CJ, Loehr L, et al (2016) Race and Sex Differences in the Incidence and Prognostic Significance of Silent Myocardial Infarction in the Atherosclerosis Risk in Communities (ARIC) Study. Circulation 133: 2141-8.

10. Kannel WB, Abbott RD (1984) Incidence and prognosis of unrecognized myocardial infarction. An update on the Framingham study. N Engl J Med 311: $1144-7$. 11. Deanfield JE, Shea MJ, Wilson RA, Horlock P, Landsheere CM, et al. (1986) Direct effects of smoking on the heart: silent ischemic disturbances of coronary flow. Am J Cardiol 57: 1005-9.

12. Shi Y, Weingarten TN, Mantilla CB, Hooten WM, Warner DO (2010) Smoking and pain: pathophysiology and clinical implications. Anesthesiology 113: 977-92. 13. Radzeviciene L, Ostrauskas R (2009) Smoking habits and the risk of type 2 diabetes: a case-control study. Diabetes Metab 35: 192-7.

14. (2010) How Tobacco Smoke Causes Disease: The Biology and Behavioral Basis for Smoking-Attributable Disease: A Report of the Surgeon General. Atlanta (GA). 